\title{
Assessing Future Expectations and the Two-Dimensional Model of Affect in an Italian Population
}

Corno, G., Molinari, G., Baños, R. M.

\begin{abstract}
Future-directed thinking has been described as part of two underlying systems that integrate dimensions of affect, motivational systems, orientation to the future, and future expectations, which are initiated at the cognitive, affective, biological, behavioral, and motivational levels. The main aim of the present study is to test the two underlying frameworks model and explore future expectations in a general Italian-speaking population ( $N=345)$. Therefore, the second aim of the present paper is to confirm the factorial structure of the Subjective Probability Task (SPT; MacLeod et al., 1996), a questionnaire designed to assess specific positive and negative orientations towards the future. Results showed that the SPT has good psychometric properties and it is a reliable instrument to assess future-directed thinking. Moreover, our findings confirmed the role of future expectancies as cognitive correlates of depression and anxiety. Differently from previous studies (Clark and Watson, 1991; MacLeod et al., 1996), our results did not confirm that depression was characterized by low positive affect. We believe this paper contributes to the understanding of future expectancies and their relation with anxiety and depression, and will help to expand the availability of an instrument to assess future directed thinking.
\end{abstract}

Keywords: Future-directed thinking, Positive affect, Negative affect, Anxiety, Depression 


\section{Introduction}

Thinking about the future is a central component of human cognition. It involves the ability to project the self forward in time in order to pre-experience an event (Atance and O'Neill, 2001). Many studies have demonstrated the relationship between future-related thinking and well-being. For instance, previous research has shown that patterns of positive future-directed thinking, such as hope and optimism, are linked to reported higher quality of life and life satisfaction (Scheier et al., 1989), less distress (e.g. Brissette et al., 2002), more adaptive behaviors, and overall higher wellbeing (Carver et al., 2010). By contrast, patterns of negative future-directed thinking, as in the case of hopelessness and pessimism, are associated with maladaptive behaviors, such as alcohol (Ohannessian et al., 1994) and substance abuse (Park et al., 1997), less persistence facing life's challenges, more avoidance coping, and poor health (Carver et al., 2010; Snyder et al., 1991). Moreover, reduced anticipation of future positive events is a defining characteristic of depression, whereas anxiety is characterized by an increase in the number of perceived negative future events (Balsamo et al., 2013; Bjärehed et al., 2010; Rief et al., 2015). Besides, expectancies have been considered a core feature of mental disorders and for that reason a focal objective of treatment (Rief et al., 2015).

In their study, MacLeod et al. (1996) tested a new model of affect. They explored if the positive and negative future expectances could be included in the tripartite model of Clark and Watson (1991). The tripartite model of anxiety and depression proposed by Clark and Watson (1991) theorizes three main factors: negative affect (NA), positive affect (PA), and arousal. According to this model, both anxiety and depression are characterized by a higher NA component; however, only depression consisting of low PA and anxiety is uniquely characterized by hyperarousal (Clark and Watson, 1991; Miloyan et al., 2014). With an exploratory factor analysis (EFA), MacLeod et al. (1996) tested a model composed by two factors: the first one characterized by anxiety, depression, NA, and expectancies for future negative events; the second factor dominated by depression (negative loading), PA (positive loading), and expectancies for future positive events. Therefore, MacLeod et al., (1996) described future-directed thinking as part of two underlying systems that integrate dimensions of affect, motivational systems, orientation to the future, and future expectations, which are initiated at the cognitive, affective, biological, behavioral, and motivational levels.

(Figure 1 about here)

Regarding affect, PA and NA have been described as two orthogonal dimensions (Clark and Watson, 1991; MacLeod et al., 1996). PA refers to pleasurable engagement and reflects the extent to which one feels enthusiastic, active and alert. NA instead refers to unpleasant engagement and reflects the extent to which one feels angry, disgusted or afraid. Both PA and NA have also been used to conceptualize anxiety and depression. Anxiety and depression are characterized by a higher NA component, whether only depression is distinguished of low PA, and anxiety is singularly characterized by hyperarousal (Clark and Watson, 1991; Miloyan et al., 2014). Referring to the motivational level, previous studies (e.g. Fowles, 1988) have shown the distinction between a punishment-driven, aversive motivational system and a reward-driven, appetitive motivational system. Specifically, McNaughton (1982) referred to the former as the behavioral inhibition system, which is linked to aversive outcomes (i.e. punishment or frustrated non-reward) and inhibits ongoing behavior when an aversive outcome is perceived as likely. By contrast, the appetitive motivational system has been called the behavioral activation system or behavioral approach system. The behavioral approach system mediates responses to signs of desirable outcomes (i.e. relieving nonpunishment or reward) and initiates approach behavior when such outcomes are perceived as probable (e.g. Fowles, 1988; Gray, 1987). Regarding future-oriented cognitive processes, they have been associated with emotional disturbances (Beck et al., 1987). Anxiety and depression are 
similarly associated with an increased tendency to anticipate the occurrence of negative events, and an increased tendency to believe that future events will yield negative outcomes (MacLeod et al., 1996; Miranda and Mennin, 2007). For instance, hopelessness has been described as the typical orientation to the future found in depression (Beck et al., 1988; MacLeod et al., 1996), whereas worry and negative bias for future personal (i.e., related to the self), but not for impersonal (i.e., other-oriented), events has been described as a characteristic of anxiety (Barlow, 1988; Butler and Mathews, 1987; MacLeod et al, 1996; Molina and Borkovec, 1994). Moreover, mood-disturbed individuals have been found to overestimate the probability of negative events (e.g. Andersen et al., 1992) and sometimes underestimate the probability of positive events (e.g. Pyszczynski et al., 1987).

To assess future-directed thinking, MacLeod et al. (1996) developed the Subjective Probability Task (SPT), a questionnaire designed to measure the tendency toward specific positive and negative future expectancies (e.g. Meevissen et al., 2011; Peters et al., 2010). Different variations and adaptations of the SPT have been used in research (e.g. Boselie et al., 2014; Hanssen et al., 2013; Stöber, 2000). The original authors developed a revised version by increasing the number of positive items (from 10 to 14) and reducing the number of negative items (from 20 to 16). Nevertheless, this change did not produce significant improvements. The original version of the SPT has been validated in English-speaking samples mostly composed by students (MacLeod et al., 1996; Meevissen et al., 2011; Peters et al., 2010).

The main aim of this study is to test the model with two underlying cognitive-affective frameworks (e.g. Clark et al., 1994; Fowles, 1988; Gray, 1987; MacLeod et al., 1996; McNaughton, 1982), in an Italian-speaking general population. To achieve this objective, we translate and assess the factorial structure and psychometric properties of the Italian SPT, a measure of future expectations, and we test the correlations between this measure and the other components of the two underlying systems (i.e. depression, anxiety, and positive and negative affect). We hypothesize that the Italian version of the SPT will present a two-factor structure and good internal consistency, like the previous version (MacLeod et al., 1996). The second objective is to test with a confirmatory factor analysis (CFA) the structure of the two-framework model, which includes future-related thinking, affect, anxiety, and depression. Based on previous outcomes (MacLeod et al., 1996), we hypothesize that analyses will reveal a two-factor structure with one factor dominated by anxiety, depression, $\mathrm{NA}$, and expectancies for future negative events, and the second factor dominated by depression (negative loading), and a positive loading of PA, and expectancies for future positive events.

\section{Methods}

\subsection{Participants}

The sample was composed of 345 participants who voluntarily took part in the present study. The sample was composed of $34.8 \%(n=120)$ men and $65.2 \%(n=225)$ women. The participant average age was 33.5 years ( $S D=12.20$.; range: $18-80$ years old). Specifically, $63.9 \%$ of subjects were between 18 to 30 years old, $11.4 \%$ between $31-40,13.2 \%$ between $41-50,10.5 \%$ between $51-60$, and $1 \%$ more than 60 years old. All participants were native Italian speakers. Regarding education, $2.6 \%$ $(n=9)$ had completed middle school, 20.3\% $(n=70)$ had finished high school, and $77.1 \%(n=266)$ reported a university level of education. Of 250 participants who filled-out the BDI-II, 21 (8.4\%) reported a moderate level of depression, and $9(3.6 \%)$ moderate-severe depression. Mean score on the BDI was $9.61 \pm 7.70$ (range: $1-39$ ). Of 256 subjects who completed the STAI-Y trait, 102 (39.84\%) scored more than 40. Mean score on the STAI-Y trait was $43.65 \pm 9.26$ (range: $26-72$ ).

\subsection{Italian Translation of the SPT}

Permission to translate and validate the SPT was granted by the authors of the instrument (MacLeod et al., 1996). First, a native Italian speaker who was aware of the purpose of the SPT translated the items from English to Italian. Second, an Italian-English bilingual speaker who was not familiar with 
the SPT performed a back-translation from Italian to English. The two English versions were compared and any discrepancies were detected. Therefore, the Italian version of the SPT was judged to be an accurate translation of the English version.

\subsection{Measures}

Positive and Negative affect. Affect was assessed with a widely used scale, the Positive and Negative Affect Scale (PANAS; Watson et al., 1988), which consists of two subscales, one measuring PA and the other measuring NA. Each subscale contains 10 items, scored on a 5-point Likert scale ranging from 1 (very slightly or not at all) to 5 (extremely). The Italian validated version of the PANAS was used in this study (Terraciano et al., 2003). The internal consistency coefficients found for the PANAS subscales in the present study were $\alpha=0.91$ for NA and $\alpha=0.90$ for PA.

Depression. Depression was assessed with the Beck Depression Inventory-II (BDI-II; Beck et al., 1996). This self-report instrument is a 21-item scale. Each item is rated on a 4-point scale (0-3). Analyzing the psychometric properties and the factor structure of the BDI-II in both analogue and clinical populations, Beck et al. (1996) found that it has good internal consistency ( $\alpha$ 's of 0.92 and 0.93, respectively) and 1-week test-retest reliability $(r=0.93)$. It has been found to be a valid indicator of depression with good diagnostic discrimination (Dozois et al., 1998). The Italian version of the BDI-II has been validated by Ghisi and colleagues (2006), which shown a satisfying internal validity (from $\alpha=0.76$ to $\alpha=0.87$ ) (Balsamo and Saggino, 2007). The internal consistency coefficient for the Italian version of the BDI-II in the present study was $\alpha=0.89$.

Anxiety. Anxiety was assessed using one of the most popular measures: the Spielberger State-Trait Anxiety Inventory- Form Y (STAI-Y; Spielberger, 1973, 2010). It consists of a brief self-report questionnaire designed to measure and differentiate between trait (a stable personality trait) and state (a temporary and fluctuating condition) anxiety. The STAI-Y consists of two subscales with 20 items each. It is a reliable and sensitive measure of anxiety ( $\alpha=0.90$ for trait scale, $\alpha=0.93$ for state scale) (Bados et al., 2010; Barnes et al., 2002; Gros et al., 2007; Spielberger, 1970). In this study, the validated Italian version of the STAI-Y trait was used (Pedrabissi and Santinello, 1989), which have shown a good internal validity ( $\alpha$ between 0.85 and 0.90) (Balsamo et al., 2013). The internal consistency coefficients found for the Italian version of the STAY-Y trait in the present study was $\alpha=$ 0.89 .

Future expectancies. Future-directed expectancies have been assessed through the Subjective Probability Task (SPT; MacLeod et al., 1996), composed of 30 items rated on a 7-point Likert scale. The participant has to estimate the probability of each item happening to him/her in the future, from 1 ("not at all likely to occur") to 7 ("extremely likely to occur"). The SPT has two subscales: one with 20 items referring to negative expectancies, and the other with 10 items referring to positive expectancies. An independent subtotal for each subscale has to be calculated. The negative expectancies subtotal score ranges from a minimum of 20 to a maximum of 140 , while the positive expectancies subtotal score ranges from a minimum of 10 to a maximum of 70 . The authors of the original version of the SPT reported that the scale has good internal consistency ( $\alpha=0.90$ for the negative subscale and $\alpha=0.86$ for the positive subscale). The two subscales also showed good discriminant validity (MacLeod et al., 1996). The internal consistency coefficients found for the Italian version of the SPT subscales in the present study were 0.92 and 0.85 for negative and positive expectancies, respectively.

\subsection{Procedure}

Participants were recruited through e-mail, social networks, and word of mouth. The survey was carried out using the Survey Monkey web platform. Demographic data (i.e. gender, age, education level, nationality, and country of residence) were collected. The translated version of the SPT and 
the Italian validated versions of the STAY-Y trait, BDI-II, and PANAS were administered, in that order. It was possible to answer to the survey in approximately 30 minutes.

\subsection{Data Analysis}

Internal consistency of the Italian SPT subscales was assessed using Cronbach's $\alpha$ coefficient. This coefficient ranges from 0 to 1 , with higher values corresponding to better reliability. The analysis assesses the correlation of each item with the subscale, as well as the change in the Cronbach's $\alpha$ coefficient if an item was excluded. Criterion validity of the Italian SPT was examined by calculating the correlation of each subscale with measures of affect, depression, and anxiety. The discriminant validity of the SPT subscales was tested by calculating item-total correlations for the positive and negative items. Construct validity of the model composed of two affect dimensions (one factor dominated by anxiety, depression, NA and expectancies for future negative events, and the second factor dominated by depression [negative loading], PA [positive loading], and expectancies for future positive events) was estimated with two confirmatory factor analyses (CFA), using the EQS program, version 6.1. First, we tested a model composed by one factor dominated by anxiety, depression, NA and expectancies for future negative events, and the second factor dominated by PA and expectancies for future positive events. Then, we tested another model with one factor dominated by anxiety, NA and expectancies for future negative events, and the second factor dominated by depression (negative loading), PA (positive loading), and expectancies for future positive events.

\section{Results}

Table 1 shows the means and standard deviations of all the measures included in the study. T tests were conducted and no significant differences related to age level were found.

(Table 1 about here)

\subsection{Confirmatory Factor Analysis of the SPT}

Initially, the factorability of the SPT items was examined. The assessment of the distribution of data showed that our data were not characterized by a normal distribution (Skewness range: -0.489 2.487; Kurtosis range: $-0.923-7.772$ ).

A CFA with the Robust Maximum Likelihood estimation method was used to test the Italian version of the SPT. First, a one-factor model (Model 1 ) was fit to the data to serve as a baseline and identify salient sources of error. This model did not fit the data well (see Table 2). Second, a model containing two correlated first-order factors of the SPT was tested. This latter model agrees with the original version of the questionnaire (MacLeod et al., 1996) (see Table 2). The fit indexes indicated that the two-factor structure of the Italian version of the SPT was a better representation than the one-factor model. As Table 3 shows, all factor loadings were above 0.40 .

(Table 2 about here)

(Table 3 about here)

\subsection{Reliability: Internal Consistency}

The internal consistency coefficients of the two subscales of the SPT were excellent (for negative expectancies $\alpha=0.92$, and for positive expectancies $\alpha=0.85)$. These results are similar to those reported by other authors (MacLeod et al., 1996; Meevissen et al., 2011; Peters et al., 2010).

\subsection{Correlation Analyses}

Correlation coefficients are presented in Table 4. All correlations were significant at $p<0.01$. Measures of negative affect, anxiety, and depression correlated positively with the negative expectancies subscale and negatively with the positive expectancies subscale, while positive affect 
correlated negatively with the negative expectancies subscale and positively with the positive expectancies subscale. The two SPT subscales were negatively correlated $(p=-0.20)$.

(Table 4 about here)

\subsection{Confirmatory Factor Analysis of the Two Affective Systems}

The factorability of the model (MacLeod et al., 1996) composed of two affect dimensions (one factor dominated by anxiety, depression, NA and expectancies for future negative events, and the second factor dominated by depression-negative loading, PA and expectancies for future positive events) was estimated by confirmatory factor analysis (CFA). The Kaiser-Meyer-Olkin (KMO) test of sampling adequacy showed that the factor model was appropriate (0.800). Additionally, Bartlett's test of sphericity was significant $\left(x^{2}(15)=776.821 ; p<0.00\right)$, revealing the data's suitability for a CFA. Two models containing two correlated first-order factors were tested. Model 1 agrees with the EFA results of MacLeod et al. (1996) in terms of one factor dominated by anxiety, depression, NA and expectancies for future negative events, and the second factor dominated by PA and expectancies for future positive events. The fit indexes support the two-factor structure of the model. Although almost all of the indexes show adequate fit, it should be noted that the RMSEA value is not adequate, as it is $>.08$ (see Table 5). As Table 6 shows, all factor loadings were excellent and above .65. In Model 2, we tested the model sustained by Clark and Watson and by the EFA results of MacLeod et al., according to which depression loads in the positive affect dimension. This model does not present an adequate fit (CFI and GFI<0.90; RMSEA>0.08) (see Table 5)

(Table 5 about here)

(Table 6 about here)

\section{Discussion}

The main aim of this study was to test the model with two underlying cognitive-affective frameworks, theorized and demonstrated by different authors (e.g. Clark et al., 1994; Fowles, 1988; Gray, 1987; MacLeod et al., 1996; McNaughton, 1982), in an Italian-speaking general population. In order to achieve this objective, we translated and assessed the factorial structure and psychometric properties of the Italian SPT, a measure of future expectations. In addition, we tested the correlations between this measure and the other components of the two underlying systems (i.e. depression, anxiety, and positive and negative affect).

We hypothesized that the Italian version of the SPT would present the same factor structure, psychometric characteristics, and good internal consistency as the previous version (MacLeod et al., 1996). Our findings showed that the Italian version of the SPT has good internal consistency, all items loaded in the same factor structure as the original version, and the two subscales were negatively correlated, as reported in previous versions of the questionnaire.

Moreover, the CFA showed that the factors were accurate representations of the two original subscales of the SPT: negative expectancies- NE (F1), and positive expectancies -PE (F2). Our findings also confirmed the negative correlations between the two subscales reported in the original version.

Our second hypothesis, confirm a two-factor structure with one factor dominated by anxiety, depression, NA, and expectancies for future negative events, and the second factor dominated by depression (negative loading), and a positive loading of PA, and expectancies for future positive events, has been partially confirmed. The CFA with measures of depression, anxiety, and positive and negative affect partially confirmed the model proposed by the authors (Clark and Watson, 1991; MacLeod et al., 1996) for the two cognitive-affective-motivational systems. Our results confirmed the features of the negative factor proposed by MacLeod et al. (1996), whether the positive factor has not been confirmed by our results. In the original study conducted by Macleod et al. (1996) with 
a student sample, both anxiety and depression have been associated with the negative affect system, while only depression has been associated with the positive affect system. This is in line with the tripartite model proposed by Clark and Watson (1991) that posits that anxiety and depression share a common component of negative affect, but can be differentiated by low positive affect associated with depression and high physiological hyperarousal associated with anxiety (Anderson and Hope, 2006). Although this theoretical background suggests that depression is also characterized by reduced activation of the motivational system that mediates PA, approach behavior, hope, and expectancies of positive outcomes, our findings did not confirm this perspective. Evaluations of the tripartite model have had varying results, possibly due to methodological and sampling differences (Anderson and Hope, 2008). We can hypothesize that the different result could be attributed to other factors. First, anxiety and depression are difficult to discriminate in community samples with available measures but are more easily differentiated as symptomatology reaches diagnosable levels (Cummings, Caporino, and Kendall, 2014). Second, our sample was taken from the general population with low scores on depression and high positive affect. Furthermore, another significant difference is the type of analyses conducted. We performed a CFA instead of an EFA. Exploratory factor analysis is not designed to test hypotheses or theories; it is used to explore a data set. On the contrary, confirmatory factor analysis allow researchers to test hypotheses via inferential techniques, and can provide more informative analytic options (Costello and Osborne, 2005). Lastly, the scale used to measure depression in the original study differed from the one used in the present study. In one of their two studies, Macleod et al. (1996) evaluated depression and anxiety with a self-report scale specifically designed for the study, and in the second study they included the Hospital Anxiety and Depression scale (HADS; Zigmond and Snaith, 1983). Therefore, future studies should clarify whether depression is characterized by both an increase in negative expectancies for the future and a decrease in positive expectancies, or only by the latter, using different scales to measure depression. Regarding the high root mean square error of approximation (RMSEA $=0.107$ ) reported by the CFA of the two-affect dimension model, it could be explained by the sample size. In fact, as Chen and colleagues (2008) suggested, the widely adopted cutoff value of 0.05 for this index rejects too many valid models in small sample sizes $(n \leq 100)$, while performing better in larger sample sizes (although it tends to over-accept at $n \geq 800$ ). Nevertheless, future studies should be carried out to verify our findings.

These findings about the role of future expectancies could represent a target for psychological interventions aimed to prevent depression and anxiety symptomatology. In fact, recent studies involving exercises that promote positive future thinking (e.g. best possible self; Peters et al., 2010; MacLeod et al., 2008) show that these pathway thinking can effectively increase positive affect and reduce depression, negative affect, and dysfunctional attitudes (Renner et al., 2014). Therefore, the SPT questionnaire represents a valid instrument that can assess the effectiveness of psychological interventions aimed to establish the casual relationship between optimism and various cognitive behavioral and affective correlates (Meevissen et al., 2011).

This study presents some limitations. First, it involved only the general population, whereas it would be interesting to use a clinical sample as well. Second, the present study is characterized by the lack of a test-retest reliability analysis for the Italian version of the SPT. In order to overcome this limitation, additional studies are needed to determine whether the Italian version of the SPT is a reliable instrument over time. Third, it would be interesting to analyze the role of other variables, such as worry and hopelessness, in the two underlying systems identified by the original authors, in a general and/or clinical Italian population.

There are different and, sometimes, contradictory approaches in the literature about models of affect (e.g. Denollet and De Vries, 2006). Therefore, further studies are recommended in order to investigate more about this thematic. The present study generated new findings that can feed the debate on the characterization of a model of affect. Moreover, since psychology has become an international science (Alonso-Arbiol and van de Vijver, 2010; Ziegler and Bensch, 2013), it is 
necessary to guarantee the comparability of the method of assessment used in order to be able to compare findings of researches in different languages (Ziegler and Bensh, 2013). Therefore, the present validation of the SPT in an Italian-speaking population will help to expand the availability of this instrument beyond the English- and Spanish-speaking world, creating new opportunities to conduct research with the Italian population.

\section{References}

Alonso-Arbiol, I., van de Vijver, F.J.R., 2010. A historical analysis of the European Journal of Psychological Assessment. Eur. J. Psychol. Assess. 26, 238-247.

American Psychiatric Association, 2000. Diagnostic and statistical Manual of Mental Disorders (revised 4th Ed.). Author, Washington, DC.

Andersen, S.M., Spielman, L.A., Bargh, J.A., 1992. Future-event schemas and certainty about the future: automaticity in depressives' future-event predictions. J. Pers. Soc. Psychol. 63 (5), 711-723.

Anderson, E.R., Hope, D.A., 2009. A review of the tripartite model for understanding the link between anxiety and depression in youth. Clin. Psychol. Rev. 28 (2), 275-287.

Atance, C.M., O'Neill, D.K., 2001. Episodic future thinking. Trends Cogn. Sci. 5 (12), 533-539.

Bados, A., Gomez-Benito, J., Balaguer, G., 2010. The state-trait anxiety inventory, trait version: does it really measure anxiety? J. Pers. Assess. 92 (6), 560-567.

Balsamo, M., Romanelli, R., Innamorati, M., Ciccarese, G., Carlucci, L., Saggino, A., 2013. The statetrait anxiety inventory: shadows and lights on its construct validity. J. Psychopathol. Behav. 35 (4), 475-486.

Balsamo, M., Saggino, A., 2007. Test per l'assessment della depressione nel contesto italiano: un'analisi critica. Italian Journal of Cognitive and Behavioural Psychotherapy 13 (2), 167.

Barlow, D.H., 2000. Anxiety and its disorders. Guilford Press, New York.

Barnes, L.L., Harp, D., Jung, W.S., 2002. Reliability generalization of scores on the Spielberger statetrait anxiety inventory. Educ. Psychol. Meas. 62 (4), 603-618.

Beck, A.T., Brown, G., Steer, R.A., Eidelson, J.I., Riskind, J.H., 1987. Differentiating anxiety and depression: A test of the cognitive content-specificity hypothesis. J. Abnorm. Psychol. 96, 179183.

Beck, A.T., Riskind, J.H., Brown, G., Steer, R.A., 1988. Levels of hopelessness in DSM-III disorders: A partial test of content specificity in depression. Cognitive. Ther. Res. 12 (5), 459-469.

Beck, A.T., Steer, R.A., Brown, G.K., 1996. Beck depression inventory-II. San Antonio.

Bjärehed, J., Sarkohi, A., Andersson, G., 2010. Less positive or more negative? Future-directed thinking in mild to moderate depression. Cogn. Behav. Ther. 39 (1), 37-45.

Boselie, J.J., Vancleef, L.M., Smeets, T., Peters, M.L., 2014. Increasing optimism abolishes paininduced impairments in executive task performance. Pain 155 (2), 334-340.

Brissette, I., Scheier, M.F., Carver, C.S., 2002. The role of optimism in social network development, coping, and psychological adjustment during a life transition. J. Pers. Soc. Psychol. 82 (1), 102.

Butler, G., Mathews, A., 1987. Anticipatory anxiety and risk perception. Cognitive. Ther. Res. 11, 551-565. 
Carver, C.S., Scheier, M.F., Segerstrom, S.C., 2010. Optimism. Clin. Psychol. Rev. 30 (7), 879-889.

Chen, F., Curran, P.J., Bollen, K.A., Kirby, J., Paxton, P., 2008. An empirical evaluation of the use of fixed cutoff points in RMSEA test statistic in structural equation models. Sociol. Methods. Res. $36(4), 462-494$.

Clark, D.A., Steer, R.A., Beck, A.T., 1994. Common and specific dimensions of self-reported anxiety and depression: implications for the cognitive and tripartite models. J. Abnorm. Psychol. 103 (4), 645.

Clark, L.A., Watson, D., 1991. Tripartite model of anxiety and depression: psychometric evidence and taxonomic implications. J. Abnorm. Psychol. 100 (3), 316.

Cummings, C.M., Caporino, N.E., Kendall, P.C., 2014. Comorbidity of anxiety and depression in children and adolescents: 20 years after. Psychol. Bull. 140 (3), 816.

Denollet, J., De Vries, J., 2006. Positive and negative affect within the realm of depression, stress and fatigue: The two-factor distress model of the Global Mood Scale (GMS). J. Affect. Disorders. 91 (2), 171-180.

Fowles, D.C., 1988. Psychophysiology and psychopathology: A motivational approach. Psychophysiology 25 (4), 373-391.

Ghisi, M., Flebus, G.B., Montano, A., Sanavio, E., Sica, C., 2006. Beck Depression Inventory-II. Manuale italiano. Organizzazioni Speciali, Firenze.

Gray, J.A., 1987. The psychology of fear and stress (2nd. ed.). Cambridge University Press.

Gros, D.F., Antony, M.M., Simms, L.J., McCabe, R.E., 2008. Psychometric properties of the State-Trait Inventory for Cognitive and Somatic Anxiety (STICSA): comparison to the State-Trait Anxiety Inventory (STAI). Psychol. Assessment. 19 (4), 369-381.

Hanssen, M.M., Peters, M.L., Vlaeyen, J.W., Meevissen, Y.M., Vancleef, L.M., 2013. Optimism lowers pain: Evidence of the causal status and underlying mechanisms. Pain 154 (1), 53-58.

Kagan, L.J., MacLeod, A.K., Pote, H.L., 2004. Accessibility of causal explanations for future positive and negative events in adolescents with anxiety and depression. Clin. Psychol. Psychot. 11 (3), 177-186.

MacLeod, A.K., Byrne, A., Valentine, J.D., 1996. Affect, emotional disorder, and future-directed thinking. Cogn. Emot. 10 (1), 69-86.

MacLeod, A.K., Coates, E., Hetherton, J., 2008. Increasing well-being through teaching goal-setting and planning skills: results of a brief intervention. JHS 9 (2), 185-196.

McNaughton, N., 1982. Gray's Neuropsychology of anxiety: An enquiry into the functions of septohippocampal theories. Behav. Brain. Sci. 5 (03), 492.

Meevinssen, Y., Peters, M.L., Alberts, H.J., 2011. Becoming more optimistic by imaging a best possible self: Effects of a two week intervention. J. Behav. Ther. Exp. Psychiatry 42 (3), 371-378.

Miloyan, B., Pachana, N.A., Suddendorf, T., 2014. The future is here: a review of foresight systems in anxiety and depression. Cogn. Emot. 28 (5), 795-810.

Miranda, R., Mennin, D.S., 2007. Depression, generalized anxiety disorder, and certainty in pessimistic predictions about the future. Cognit. Ther. Res. 31 (1), 71-82. 
Molina, S., Borkovec, T.D., 1994. The Penn State Worry Questionnaire: Psychometric properties and associated characteristics. In Davey, G., Tallis, F. (Eds.), Worrying: Perspectives on Theory, Assessment, and Treatment (pp. 265-283), John Wiley \& Sons. Oxford, England.

Ohannessian, C.M., Hesselbrock, V.M., Tennen, H., Affleck, G., 1994. Hassles and uplifts and generalized outcome expectancies as moderators on the relation between a family history of alcoholism and drinking behaviors. J. Stud. Alcohol. Drugs. 55 (6), 754.

Osborne, J.W., Costello, A.B., 2009. Best practices in exploratory factor analysis: Four recommendations for getting the most from your analysis. PPMR 12 (2), 131-146.

Park, C.L., Moore, P.J., Turner, R.A., Adler, N.E., 1997. The roles of constructive thinking and optimism in psychological and behavioral adjustment during pregnancy. J. Pers. Soc. Psychol. $73(3), 584$.

Pedrabissi, L., Santinello, M., 1989. Inventario per l'ansia di «Stato» e di «Tratto»: nuova versione italiana dello STAI Forma Y: Manuale. Organizzazioni Speciali, Firenze.

Peters, M.L., Flink, I.K., Boersma, K., Linton, S.J., 2010. Manipulating optimism: Can imagining a best possible self be used to increase positive future expectancies? J. Posit. Psychol. 5 (3), 204-211.

Pyszczynski, T., Holt, K., Greenberg, J., 1987. Depression, self-focused attention, and expectancies for positive and negative future life events for self and others. J. Pers. Soc. Psychol. 52 (5), 994.

Renner, F., Schwarz, P., Peters, M.L., Huibers, M.J., 2014. Effects of a best-possible-self mental imagery exercise on mood and dysfunctional attitudes. Psychiat. Res. 215 (1), 105-110.

Rief, W., Glombiewski, J.A., Gollwitzer, M., Schubö, A., Schwarting, R., Thorwart, A., 2015. Expectancies as core features of mental disorders. Curr. Opin. Psychiatry. 28 (5), 378-385.

Scheier, M.F., Matthews, K.A., Owens, J.F., Magovern, G.J., Lefebvre, R.C., Abbott, R.A., Carver, C.S., 1989. Dispositional optimism and recovery from coronary artery bypass surgery: the beneficial effects on physical and psychological well-being. J. Pers. Soc. Psychol. 57 (6), 1024.

Snyder, C.R., Irving, L.M., Anderson, J.R., 1991. Hope and health. Handbook of social and clinical psychology: The health perspective, 162, 285-305.

Spielberger, C.D., Gorsuch, R.L., Lushene, R.E., 1970. Manual for the State-Trait Anxiety Inventory. Consulting Psychologists Press, Palo Alto, CA.

Spielberger, C.D., 1973. STAIC preliminary manual. Consulting Psychologists Press, Palo Alto, CA.

Spielberger, C.D., 2010. Test anxiety inventory. John Wiley \& Sons, Inc.

Stöber, J., 2000. Prospective cognitions in anxiety and depression: Replication and methodological extension. Cogn. Emot. 14 (5), 725-729.

Terraciano, A., McCrae, R.R., Costa Jr., P.T., 2003. Factorial and construct validity of the Italian Positive and Negative Affect Schedule (PANAS). Eur. J. Psychol. Assess. 19 (2), 131.

Watson, D., Clark, L.A., Tellegen, A., 1988. Development and validation of brief measures of positive and negative affect: the PANAS scales. J. Pers. Soc. Psychol. 54 (6), 1063.

Ziegler, M., Bensch, D., 2013. Lost in translation: Thoughts regarding the translation of existing psychological measures into other languages. Eur. J. Psychol. Assess. 29 (2), 81-83.

Zigmond, A.S., Snaith, R.P., 1983. The hospital anxiety and depression scale. Acta. Psychiat. Scand. 67 (6), 361-370. 
